\title{
Ein aktuelles Kompendium über die Saamen und ihre Kultur
}

Saamentutkimus tänään [Saamische Studien heute]. Hrsg. v. IRJA Seurujärvi-Kari, Petri HaLinen und Risto Pulkkinen. Tietolipas 234. Suomalaisen Kirjallisuuden Seura, Helsinki 2011. 449 S.

Die Saamischen Studien sind in den letzten Jahren sowohl in Finnland als auch in Schweden und Norwegen reger geworden. Heute sind auch gebürtige Saamen unter den Vertretern des Fachs. Die Wissenschaftler, die sich an der Universität Helsinki mit der saamischen Sprache und Kultur befassen, haben gemeinsam mit ihren skandinavischen Kollegen in den letzten zwei Jahrzehnten drei Sammelbände vorgelegt, die vielseitige Informationen über Sprache, Geschichte, Kultur, Lebensbedingungen und gesellschaftliche Stellung des indigenen Volkes im Norden bieten. Der erste, Johdatus saamentutkimukseen [Einführung in die Saamischen Studien] (hrsg. v. Ulla-Maija Kulonen, Juha Pentikäinen und Irja SeurujärviKari; Tietolipas 113, Helsinki 1994) enthielt grundlegende Informationen über die saamische Kultur und ihre Entstehung. Der im Jahr 2000 unter der Nummer 164 ebenfalls in der Reihe Tietolipas erschienene Band Beaivvi mánát. Saamelaisten juuret ja nykyaika [Kinder der Sonne. Die Wurzeln der Saamen und die Gegenwart] (hrsg. v. Irja Seurujärvi-Kari) beruhte auf einer Reihe von Seminaren, die in den Jahren 1996-98 stattfanden und die Rechte der indigenen Völker vornehmlich aus der Sicht der Saamen behandelten. Gemeinsam bilden diese beiden Publikationen und der hier zu besprechende Band Saamentutkimus tänään ein solides Informationspaket für alle, die sich für die saamische Kultur und generell für indigene Völker interessieren.

Saamentutkimus tänään enthält 14 Beiträge zur Sprache, Kultur und Geschichte der Saamen. Die meisten Beiträge bieten nützliche Hintergrundinformationen, durch die der saamische Aspekt mit dem weiteren Rahmen des jeweiligen Themas verknüpft wird. Die rechtliche Stellung der Saamen und der indigenen Völker überhaupt wird in drei Artikeln behandelt. Irja Seurujärvi-Kari betrachtet die Beziehung der Saamen zur Bewegung der indigenen Völ- 
ker. Die Definition der saamischen Nationalität beruht in Skandinavien auf einem subjektiven und einem objektiven Kriterium. Unter dem subjektiven Kriterium ist die Frage zu verstehen, $o b$ eine Person sich für saamisch hält oder nicht. Das objektive Kriterium ist die saamische Sprache, die die Person selbst oder ein Eltern- oder Großelternteil als erste Sprache erlernt haben muss. Die Anzahl der Saamen in Finnland wird auf 9 ooo - 10 ooo geschätzt. Im sog. Saamengebiet, d. h. in den nördlichsten Kommunen Finnlands, werden die sprachlichen Rechte der Saamen relativ gut verwirklicht, doch $60 \%$ der Saamen leben außerhalb dieses Gebiets. In den Nachbarländern Finnlands stellt sich die Situation anders dar: In Schweden leben $20 \%$ und in Norwegen nur $10 \%$ der Saamen außerhalb des saamischen Gebiets. Saamen sind schon seit den 1970er Jahren in der Bewegung der indigenen Völker aktiv.

Nach Ansicht des Rechtswissenschaftlers Timo Koivurova wird das Selbstverständnis der saamischen Kultur durch die Menschenrechte generiert: Da man die Forderungen nicht nach den Spielregeln der Nationalstaaten, die auf der Mehrheitsdemokratie basieren, durchsetzen konnte, berief man sich auf die internationalen Menschenrechtsabkommen. Zwar sind die Menschenrechte ein Teil der Gesamtheit des internationalen Rechts, doch werden sie in der Regel durch das nationale Rechtssystem verwirklicht. Das Selbstbestimmungsrecht eines indigenen Volkes legitimiert nicht die Loslösung vom Mutterland, sondern allenfalls eine Art Autonomie oder Selbstverwaltung. Beispielsweise können die von den Saamen angestrebten Landbesitz- und Bodennutzungsrechte im Widerspruch zu den Gesetzen der betreffenden Staaten und zum Schutz des persönlichen Eigentums stehen. In ihrem Beitrag über den Vertrag 169 der ILO über die indigenen Völker betrachtet Tanja Joona diese Frage aus der Sicht der finnischen Saamen.

Der Biologe Tuomas Heikkilä und der Ökologe Antero Järvinen stellen in ihrem Beitrag Saamelaisalueen luonto ja sen muutokset [Die Natur des saamischen Gebiets und ihre Veränderungen] die Verhältnisse im Norden und die eventuellen Auswirkungen des Klimawandels dar. Den indigenen Völkern wird häufig ein Leben im Einklang mit der Natur attestiert, doch Heikkilä und Järvinen zeigen auf, dass auch sie die Natur ebenso kurzsichtig und intensiv ausbeuten können wie andere Völker, sofern sie über die finanziellen und technologischen Mittel verfügen. Die Hauptkritik der Verfasser richtet sich gegen die Überweidung in der Rentierzucht und den Hass der Saa- 
men auf Raubtiere. Antti Sajantila befasst sich mit dem genetischen Hintergrund der Saamen.

Der Beitrag Saamelaiskielet nykypäivää ja historiaa [Die saamischen Sprachen - Gegenwart und Geschichte] von Janne Saarikivi ist eine hervorragende Einführung in die saamischen Sprachen, ihre Entwicklung und ihre Verwandtschaftsbeziehungen. Saarikivi betrachtet zunächst das Verhältnis zwischen Dialekt und Sprache und stellt fest, dass die dialektale Gliederung des Saamischen traditionell auf phonetischen und morphologischen Kriterien beruht. Die Konzentration auf den Wortschatz würde vermutlich $\mathrm{zu}$ einer anderen Gliederung führen, die zugleich die kulturellen Einflüsse widerspiegeln würde. Der Verfasser erörtert die Expansion der einzigen großen saamischen Sprache, des Nordsaamischen (das von $80 \%$ aller Saamen gesprochen wird); sie entstand durch die in großem Maßstab betriebene Rentierzucht, die sich gegen Ende des Mittelalters in dem Gebiet verbreitete. Wichtig für die heutige sprachliche Vitalität war die nordsaamische Rechtschreibreform von 1978, der es zu verdanken ist, dass die Sprache in allen skandinavischen Ländern nach denselben Prinzipien geschrieben wird. Saarikivi zufolge schuf dies die Grundlage für die Entstehung der saamischen Hochkultur und für die heutige - im internationalen Maßstab seltene - Situation, in der das Nordsaamische in allen Funktionen der modernen Gesellschaft verwendet wird, obwohl die Zahl seiner Sprecher nur bei einigen Zehntausend liegt. Der Verfasser beschreibt die Beziehung zwischen dem Finnischen und den saamischen Sprachen sowie ihre gemeinsame und separate Geschichte. Die komprimierte Darstellung ist zugleich eine klar formulierte Einführung sowohl in die Sprachverwandtschaft und ihre Beschreibung als auch in die wichtigsten Besonderheiten des Saamischen. Flexionstabellen und Wortlisten dienen der Veranschaulichung. Saarikivi behandelt auch die lexikalischen Schichten der saamischen Sprachen, u. a. die aus unbekannten Sprachen übernommenen Substratwörter. In seinem Entwurf siedelt er das Ursaamische im Binnenland in der Gegend des Ladoga-Sees an und datiert es auf den Beginn unserer Zeitrechnung.

Als Hintergrund zu den behandelten Themen werden in den Beiträgen des Bandes die Methoden der jeweiligen Untersuchung oder Disziplin skizziert, mit denen die dargestellten Ergebnisse erreicht wurden. Petri Halinen bietet in seinem Beitrag Arkeologia ja saamentutkimus [Die Archäologie und die Saamischen Studien] zunächst grundlegende In- 
formationen über die verfügbaren Datierungsmethoden und die mit ihnen verbundenen Probleme und erörtert dann die komplizierte Beziehung zwischen archäologischen Phänomenen und Ethnizität: Wie kann man Schlussfolgerungen über die Ethnie der Vertreter einer prähistorischen Kultur ziehen, obwohl Sprache, Gene und materielle Kultur nicht unbedingt übereinstimmen? Halinen kartiert die Bevölkerungsbewegungen in Finnland und im nördlichen Fennoskandien nach der Eiszeit und stellt die Entwicklung der Gewerbe und Siedlungen von der vorgeschichtlichen Zeit bis in die Gegenwart dar. Er beschreibt auch, wie Phänomene aus dem Bereich der geistigen Kultur, z. B. des Glaubens, im Licht archäologischer Funde untersucht werden können und was Funde aus dem Bereich der materiellen Kultur über die Kultur der Saamen aussagen.

Lars Ivar Hansen polemisiert gegen die historische Forschung über die Saamen. Seiner Ansicht nach entstand die saamische Identität in der Begegnung mit den heterogenen Gemeinschaften im Norden, und die Geschichte der Saamen wurde u. a. durch die Nationalstaaten und die von ihnen verursachten Grenzverschiebungen sowie in den letzten Jahrhunderten durch die auf die Eingliederung und Nihilierung der indigenen Bevölkerung abzielende
Politik beeinflusst. Hansen betrachtet die wichtigsten von den Saamen selbst verfassten Schilderungen der Geschichte ihres Volkes. Er beschreibt den wissenschaftstheoretischen Wandel im 19. Jahrhundert, in dessen Folge eine Zweiteilung zwischen den westlichen sog. dynamischen Gesellschaften und den primitiven Gemeinschaften entstand: die ersteren wurden mit den Methoden der Geschichtswissenschaft untersucht, die letzteren der Ethnografie zugeordnet. In den jungen Nationalstaaten wurde die Geschichte $\mathrm{zu}$ einem zentralen Instrument der Nationbildung. Diese Zweiteilung ist in der Finnougristik heute noch sichtbar: Mit Finnland, Estland und Ungarn befasst sich die Geschichtsschreibung, während z. B. über die uralischen Völker in Russland vorwiegend ethnografische Darstellungen veröffentlicht wurden.

Der 6o Seiten umfassende Beitrag Saamelaisten etninen uskonto [Der ethnische Glaube der Saamen] von Risto Pulkkinen ist eine kompakte und präzise Synthese der zentralen Eigenheiten und Inhalte des vorchristlichen Glaubens der Saamen. Pulkkinens kohärente Darstellung umfasst die Entwicklung vom schamanistischen Glauben der Jäger- und Fischerkultur zur verborgenen Tradition, die in der Erzähltradition immer noch sichtbar ist, aber heute einerseits zum Bildschatz 
der Märchen wird und sich andererseits durch die Revitalisierung zu Symbolen der saamischen Kultur entwickelt, die beispielsweise in der Kunst verwendet werden. Pulkkinen vergleicht den ethnischen Glauben der Saamen sowohl mit der Tradition der verwandten arktischen Völker als auch mit der christlichen Tradition, die bereits vor dem Beginn der Missionierung Einfluss auf die saamische Mythologie hatte. Man könnte zahlreiche interessante Beobachtungen und Interpretationen hervorheben. Da sie sich zu einer hervorragenden Gesamtheit verbinden, kann ich die Lektüre des Beitrags jedem empfehlen, der sich für die saamische Kultur interessiert.

Irja Seurujärvi-Kari hat für den vorliegenden Band auch einen Beitrag über die saamische Kunst verfasst. Ursprünglich beruhte die Kunst auf der saamischen Handarbeitstradition (duodji); erst im 20. Jahrhundert begannen sich die verschiedenen Kunstgattungen zu entwickeln. Seurujärvi-Kari behandelt kursorisch die wichtigsten Vertreter der saamischen bildenden Kunst, der Literatur, des Theaters, der modernen Musik und des Films. Wie Seurujärvi-Kari betrachtet auch Gunvor Guttorm die saamische Handarbeit, duodji, als Teil der saamischen Selbstdefinition und der künstlerischen Darstellung. Sie erörtert die Rolle der Objekte als Vermittler und Quellen der Überlieferung. Helena Ruotsala stellt die materielle Kultur der Saamen vor, u. a. die Botschaften der saamischen Tracht und die ganzheitliche Bedeutung des Rentiers sowohl für das Erwerbsleben wie als Rohmaterial. Die Folkloristin Marjut Huuskonen erörtert die Beziehung der Saamen zur Natur, die sich sowohl in der erzählenden Prosa als auch in den Redewendungen widerspiegelt. Den roten Faden vieler Erzählungen bildet die Schilderung der Wechselwirkung zwischen Mensch und Umwelt; ein Ort dient als Fixpunkt des Gedächtnisses. Huuskonen betrachtet die Prosaüberlieferung aus der Sicht des eigenen Traditionsgattungssystems der Saamen.

Laut Minna Riikka Järvinen ist die saamische Musik ihrem Typ nach vokal, wird also ohne Instrumente aufgeführt. Sie kann in zwei geografische Gruppen gegliedert werden, eine westliche und eine östliche, deren Grenze am östlichen Rand des Inari-Sees verläuft. Typisch für die erstere sind Pentatonik und eine geringe Menge an Text, für die letztere dagegen epische Texte und eine lückenlose Skala. Järvinen stellt die wesentlichen Züge beider Gruppen kurz vor.

Saamentutkimus tänään ist ein vielseitiges und hochrangiges, elementares Werk der Saamischen Studien, das auch Lesern zugänglich gemacht werden sollte, die des 


\section{Márta Csepregi}

Finnischen nicht mächtig sind. Es band ist umfassender und gründlibeleuchtet alle Aspekte des Saamen- cher als seine Vorgänge, ein Werk, tums, von Geschichte und Sprache das man schon jetzt als Klassiker bis zu Kultur und Gesetzgebung. bezeichnen darf.

Die Beiträge stützen sich auf die neueste Forschung. Der Sammel-

Sirkka Saarinen 